\title{
Author Correction: Scalable synthesis of hierarchically structured carbon nanotube- graphene fibres for capacitive energy storage
}

Dingshan Yu D , Kunli Goh (D), Hong Wang, Li Wei, Wenchao Jiang, Qiang Zhang (D), Liming Dai (iD) and Yuan Chen (D)

Correction to: Nature Nanotechnology https://doi.org/10.1038/nnano.2014.93, published online 11 May 2014.

In the Supplementary Information for this Article, Supplementary Fig. 9b wrongly showed the electron energy-loss spectroscopy (EELS) spectrum for the carbon nanotube sample SWCNT-EDA instead of for the graphene sample G-EDA. The inset is correct. The original and corrected version of Supplementary Fig. $9 \mathrm{~b}$ is shown below. The corrected Supplementary Information file is attached to this correction notice; the original file has not been amended.

Original

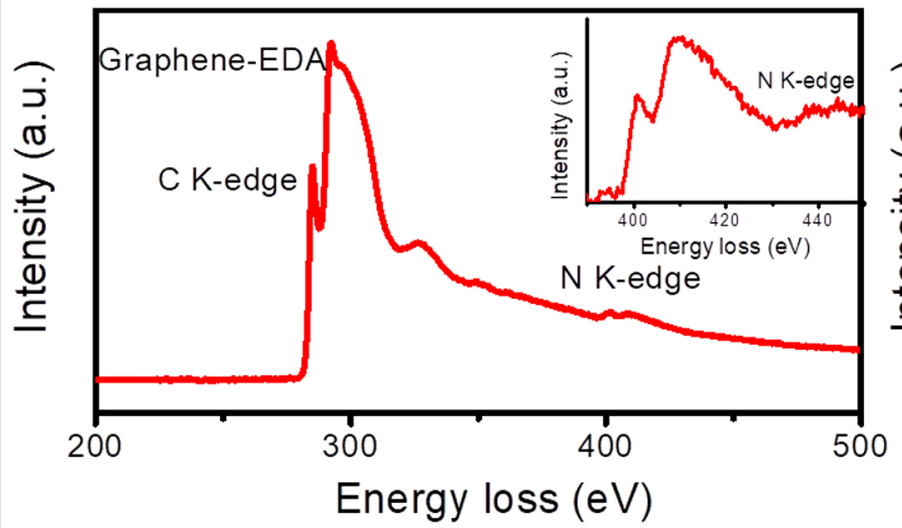

Correct

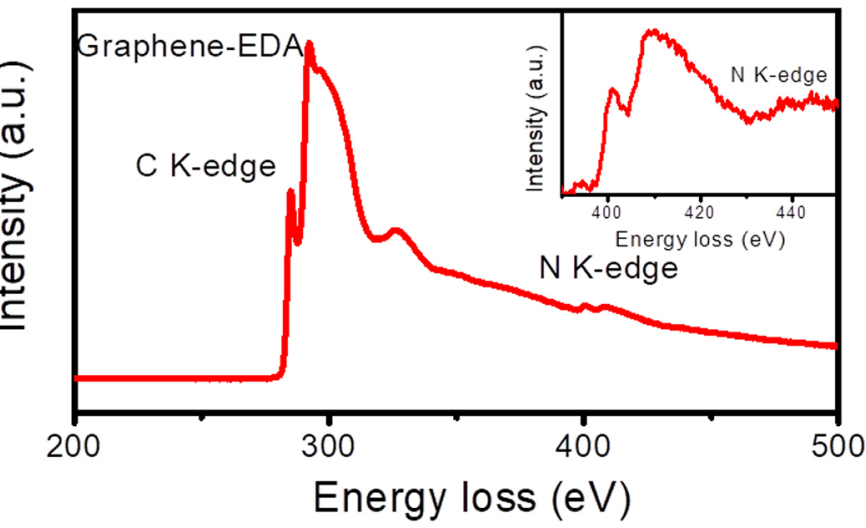

Supplementary Fig. 9b | Original and corrected.

\section{Additional information}

Supplementary information is available for this paper at https://doi.org/10.1038/s41565-020-0718-1.

Published online: 30 June 2020

https://doi.org/10.1038/s41565-020-0718-1

(c) The Author(s), under exclusive licence to Springer Nature Limited 2020 